\title{
Hubert Carrier, Le Labyrinthe de l'Etat. Essai sur le débat politique en France au temps de la Fronde (1648-1653)
}

Antonella Amatuzzi e 120-122

\section{(2) OpenEdition \\ Journals}

Edizione digitale

URL: http://journals.openedition.org/studifrancesi/29891

DOI: $10.4000 /$ studifrancesi.29891

ISSN: 2421-5856

\section{Editore}

Rosenberg \& Sellier

\section{Edizione cartacea}

Data di pubblicazione: 1 avril 2006

ISSN: 0039-2944

\section{Notizia bibliografica digitale}

Antonella Amatuzzi e 120-122, «Hubert Carrier, Le Labyrinthe de l'Etat. Essai sur le débat politique en France au temps de la Fronde (1648-1653)», Studi Francesi [Online], 148 (XLX | I) | 2006, online dal 30 novembre 2015, consultato il 19 avril 2021. URL: http://journals.openedition.org/studifrancesi/29891 ; DOI: https://doi.org/10.4000/studifrancesi.29891

Questo documento è stato generato automaticamente il 19 avril 2021.

\section{(†)

Studi Francesi è distribuita con Licenza Creative Commons Attribuzione - Non commerciale - Non opere derivate 4.0 Internazionale. 


\section{Hubert Carrier, Le Labyrinthe de l'Etat. Essai sur le débat politique en France au temps de la Fronde (1648-1653)}

Antonella Amatuzzi e 120-122

\section{NOTIZIA}

Hubert Carrier, Le Labyrinthe de l'Etat. Essai sur le débat politique en France au temps de la Fronde (1648-1653), Paris, Champion, Bibliothèque d'histoire moderne et contemporaine, 14, 2004, $694 \mathrm{pp}$.

1 Grazie ai precedenti lavori di Hubert Carrier sappiamo che cosa "sono" le Mazarinades in quanto phénomène de presse (La presse de la Fronde (1648-1653): Les Mazarinades, vol. I, La Conquête de l'opinion, Genève, Droz, 1989 - cf. questi «Studi», 100, gen.-apr. 1990, pp. 131-132 ; vol. II, Les hommes du livre, Genève, Droz, 1991 - cf. questi « Studi », 111, sett.dic. 1993, pp. 596-597). Sappiamo inoltre che cosa "dicono" dal punto di vista letterario e come si inseriscono nel contesto della produzione della metà Seicento (Les muses guerrières. Les Mazarinades et la vie littéraire au milieu du XVII siècle, Paris, Klincksieck, 1996 - cf. questi «Studi», 123, set.-dic. 1997, p. 568).

2 Comprendiamo meglio ora attraverso questa nuova fatica quali sono le idee politiche che le Mazarinades "veicolano".

3 L'autore si basa infatti su questi scritti per analizzare il dibattito politico che si aprì nella società d'oltralpe durante la Fronda. Egli intende ripercorrere gli interrogativi e i drammi che tormentarono i Francesi quando si trovarono di fronte ad un apparato statale completamente scardinato, ad un "labyrinthe de l'Etat" (così lo definisce una mazarinade del 1652 da cui Carrier prende in prestito il titolo) all'interno del quale si sentivano rinchiusi. 
4 L'autore è ben consapevole delle riserve che possono essere mosse sulla legittimità di utilizzare per uno studio sulle controversie politiche dei testi per lo più anonimi e che sono spesso espressione non dell'opinione pubblica ma di una strategia polemica che mira a manipolare questa opinione. Egli è tuttavia convinto, e a giusto titolo, che l'apporto delle Mazarinades alla conoscenza del pensiero politico e sociale dell'epoca sia preziosissimo. Si tratta infatti di documenti unici che, a condizione di essere valutati e giudicati con la dovuta prudenza, consentono di sondare le impressioni, i commenti e i pensieri dei protagonisti della Fronda e di farlo "à chaud", giorno per giorno, sul campo, assicurando così agli studiosi delle teorie politiche, della storia economica $\mathrm{e}$ sociale e della storia delle mentalità una varietà di voci e di punti di vista straordinaria.

In più l'indagine di Carrier ha il merito di non limitarsi a prendere in considerazione la produzione pamphlettistica (impresa già di per sé titanica a causa della vastità e della difficile reperibilità di questi documenti) ma di metterla spesso a confronto con altri scritti politici contemporanei (corrispondenze, mémoires, gazettes, cahiers de doléances, nouvelles di vario genere).

Questa documentazione, così ampia e frammentaria, non era stata prima d'ora oggetto di analisi approfondite ed articolate ed è questo il grandissimo pregio di questo lavoro.

7 La prima parte, "Les courants de la pensée politique", inizia col passare in rassegna quali furono gli atteggiamenti dei contemporanei nei confronti del re e delle altre persone della famiglia reale quali traspaiono dalla lettura delle Mazarinades. L'attaccamento leale, la devozione e l'idolatria della popolazione verso il giovane Luigi XIV restano ben solidi anche nel bel mezzo delle lotte civili. Diverse invece le posizioni assunte verso la regina, che viene pubblicamente odiata perché colpevole della passione cieca per Mazzarino, e verso Gaston d'Orléans che pur godeva di un certo rispetto da parte dei contemporanei in quanto «il incarnait dans sa personne hésitante une capitale tiraillée entre la violence de Condé et la haine du Mazain, un royaume déchiré entre un immense désir de paix et une aversion non moins forte pour le despotisme, et que l'opinion publique se reconnaissait dans cette insurmontable dualité» (p. 71).

8 Vengono poi esplicitate le tendenze che emergono dai documenti esaminati riguardo alle questioni politiche fondamentali. Ecco le più importanti.

9 - Quale regime è meglio privilegiare? Repubblica o monarchia? I 'frondeurs' dimostrano uno spirito sostanzialmente conservatore e restano fedeli al sistema monarchico anche quando in Inghilterra i rivoluzionari di Cromwell uccidono il re Carlo I. Vengono tutt'al più auspicati dei cambiamenti dinastici (in favore di Condé).

10 - Quanto e quale potere deve avere il popolo? Vi è la consapevolezza del ruolo politico che rivestì il popolo in occasione della journée des barricades; è altrettanto chiaro che, più tardi, Condé non avrebbe mai potuto mantenere il controllo sulla capitale senza il sostegno del popolo. Tuttavia questo sostegno è percepito come fragile, incerto e pericoloso e il popolo, considerato poco affidabile, viene piuttosto manipolato dai 'frondeurs' e discreditato da una 'campagna stampa' governativa contro "l'impertinente populace".

11 - Quali devono essere i limiti e la natura dell'autorità reale, in particolare durante il periodo della reggenza? Il parere dei contemporanei della Fronda è che si debbano mettere dei limiti all'autorità reale tornando al sistema politico precedentemente esistente in cui i corpi intermediari (Parlamento, assemblee del clero, assemblea della 
nobiltà...) temperavano le decisioni dei monarchi. Questo equilibrio è stato secondo loro spezzato dal comportamento degli ultimi ministri che hanno imposto un centralismo ed un assolutismo del tutto estranei alla tradizione monarchica francese. Negli scritti della Fronda ci si interroga quindi su temi politici capitali tra i quali la legittimità della rivolta contro i tiranni, la sovranità popolare ed altri. Si riflette anche sull'indebolimento del potere politico durante la reggenza e alcuni suggeriscono che in caso di minore età del sovrano il potere debba rimanere nelle mani dei principi di sangue e del Parlamento.

- Come devono funzionare le istituzioni? Il primo problema sollevato dagli autori delle Mazarinades è quello del ruolo eccessivo illegittimamente assunto dal Primo Ministro, per di più straniero e ecclesiastico. Solo gli scritti di provenienza 'mazzarina' giudicano l'intrusione del Cardinale indispensabile per aiutare il re ad affrontare impegni complessi e delicati. Mentre i 'frondeurs' criticano il ruolo politico esercitato dagli ecclesiastici e gli intrighi dei prelati di corte, essi ripongono nel Parlamento la loro fiducia. Questa istituzione dovrebbe controllare gli abusi della monarchia (destituendo anche se necessario i ministri) ma la sua scarsa rappresentatività e la sua decisione di appoggiare i Principi fa svanire ogni speranza frondista. Nemmeno il ricorso agli Stati Generali, giustificato dalla drammaticità della situazione e richiesto due volte durante la Fronda, troverà seguito.

13 - Quali scelte deve compiere la Francia in materia di politica estera? Gli autori delle Mazarinades si preoccupano dei rapporti internazionali della Francia, delle sue alleanze con i paesi stranieri e danno largo spazio alle notizie provenienti dall'estero. Viene rimproverato a Mazzarino di aver provocato l'isolamento del paese e sacrificato la pace dell'Europa al suo proprio interesse. Si nota poi l'emergenza di una forma di coscienza nazionale contro i nemici Spagnoli, Inglesi e Turchi.

Dalla seconda parte, "Problèmes économiques et questions fiscales", emerge come le Mazarinades denuncino gli eccessi e gli abusi del sistema fiscale, in particolare i metodi di esazione delle imposte che si sono diffusi e la moltiplicazione dei dazi e delle tasse sulle merci che hanno paralizzato il commercio. Il potere assoluto e dispotico del re in campo fiscale viene messo in discussione; tuttavia, benché si auspichi una parità della pressione fiscale, non si chiede sistematicamente la soppressione dei privilegi della nobiltà e del clero e non si contestano le esenzioni di cui essi godono. Si registra poi alla lettura delle Mazarinades una evoluzione della posizione dei 'frondeurs' per quanto concerne la politica economica. Dapprima piuttosto 'protezionisti' essi passano ad accettare il principio del libero scambio dopo il 1650.

Alcuni libelli avanzano anche delle proposte di riforma e offrono valide analisi in campo budgetario e monetario. Qualcuno arriva a stimare le cifre del budget statale e a calcolarne il deficit e l'ammontare dell'appropriazione indebita' perpetrato ai danni della nazione. La pratica di esportare i capitali all'estero è aspramente condannata e Mazzarino, l'accusato principale, è preso di mira in scritti in cui le cifre e le immagini vengono retoricamente amplificate.

16 Le Mazarinades si rivelano insomma una testimonianza inequivocabile di come i problemi economici e finanziari siano usati a scopi di propaganda politica e soprattutto sono «la première prise de conscience collective en France des problèmes économiques et financiers d'un état moderne» (p. 472).

17 La terza parte, «Structures et mentalités sociales», mostra come i modelli di società che si delineano dalla lettura delle Mazarinades non siano innovativi ma ricalchino quelli 
dei secoli precedenti. Le strutture fondamentali della società divisa in ordini, fortemente gerarchizzata, basata su legami clientelari, non vengono mai messe in questione poiché si continua a pensare che la differenza sociale sia insita nella natura delle cose, nell'ordine del mondo voluto dal Creatore. Non si immagina una grande mobilità sociale e una evoluzione delle varie istituzioni, non vi sono rivendicazioni di classe unitarie, ma si assiste invece a divisioni profonde all'interno dei diversi ordini (clero secolare contro 'haut clergé', nobiltà parigina contro nobiltà 'proletaria' delle province, ricchi mercanti borghesi e piccola borghesia). Le richieste di eguaglianza sociale sono rare. Si riscontra però il concetto di 'mutualité sociale': di fronte al dramma che vive la nazione si pone attenzione alle ingiustizie sociali e alle situazioni di miseria e si sente la necessità di intervenire con azioni di solidarietà e assistenza reciproca.

18 Tirando le fila della sua analisi, Carrier afferma tra l'altro che dalla lettura dei testi dell'epoca risulta chiaramente come l'insuccesso della Fronda, "rétrograde dans le domaine politique, franchement réactionnaire sur les questions économiques et fiscales, (...) fixiste sur le plan social» (p. 602) fu dovuto all' «absence d'un véritable programme de réformes emportant l'adhésion de tous les opposants, d'un projet fédérateur susceptible de convaincre les intelligences et de rassembler durablement les énergies» (p. 604).

A noi non resta che rallegrarci per questa ulteriore opera di Hubert Carrier che rende meno arduo muoversi nell'intricato "labirinto" ideologico e politico della Fronda e si configura come un contributo indispensabile per chi voglia avventurarsi nello studio delle idee politiche dell'Ancien Régime.

Aggiungiamo ancora che il ricco apparato di note che correda il testo rappresenta uno strumento validissimo, fornendo, tra l'altro, i rimandi a tutti i numerosissimi documenti frequentemente e puntualmente citati. Molto utili anche la cronologia dei tormentati avvenimenti della Fronda e la ricca bibliografia. 